\title{
The Endogenous Vascular Elastase That Governs Development and Progression of Monocrotaline-induced Pulmonary Hypertension in Rats Is a Novel Enzyme Related to the Serine Proteinase Adipsin
}

\author{
Li Zhu, * Dennis Wigle, * Aleksander Hinek, * Jun Kobayashi, * Chongliang Ye, * Marc Zuker, * Hidemi Dodo, * \\ Fred W. Keeley, ${ }^{\star}$ and Marlene Rabinovitch* \\ Division of Cardiovascular Research, Research Institute, The Hospital for Sick Children, and Departments of *Pediatrics and Pathology \\ and ${ }^{\ddagger}$ Biochemistry, University of Toronto, Toronto, Ontario, Canada M5G 1X8
}

\begin{abstract}
We showed previously a cause and effect relationship between increased activity of an endogenous vascular elastase (EVE) and experimentally induced pulmonary hypertension in rats. We now report the isolation and characterization of EVE. Degenerate oligonucleotides synthesized to homologous sequences in serine elastases were used in a PCR with rat pulmonary artery (PA) cDNA. The PCR product hybridized to a 1.2-kb mRNA and the intensity of hybridization was threefold increased in RNA from rat hypertensive PA at a timepoint when EVE activity was increased. The PCR product was used to screen a cDNA library and sequences obtained encoded rat adipsin. We then used immunoaffinity to purify EVE. An antibody to the elastin-binding protein was used to remove this competitor of elastase from the PA extract and the elastolytic activity increased 100fold. The enzyme was purified using an antibody that recognizes $\mathrm{NH}_{2}$-terminal sequences of serine proteinases and the eluate was further purified using an antibody raised against recombinant adipsin. A single band at $20 \mathrm{kD}$ immunoreactive with the adipsin antibody was resolved as an active enzyme on an elastin substrate gel. Immunogold labeling with an antibody to an adipsin peptide sequence localized EVE to PA smooth muscle cells. This is the first isolation of EVE; it appears to be a novel enzyme related to the serine proteinase adipsin originally found in adipose tissue. ( $J$. Clin. Invest. 1994. 94:1163-1171.) Key words: elastin • vascular disease - vascular smooth muscle • pulmonary artery
\end{abstract}

\section{Introduction}

Pulmonary hypertension, the result of progressive vascular changes in the pulmonary arteries (PA), ${ }^{1}$ is a common and

Portions of this work were published in abstract form (1992. FASEB [Fed. Am. Soc. Exp. Biol.] J. 7:1033a) (1993. Mol. Biol. Cell. 4[Suppl.]:410a).

Address correspondence to Marlene Rabinovitch, M.D., Division of Cardiovascular Research, The Hospital for Sick Children, 555 University Avenue, Toronto, Ontario M5G 1X8, Canada.

Received for publication 10 March 1994.

1. Abbreviations used in this paper: anti-S-Gal, antibody to the elastin binding domain of the spliced variant of $\beta$ galactosidase; $\mathrm{BCZ}$, antibody recognizing the lectin site of EBP; EBP, elastin binding protein; EVE, endogenous vascular elastase; HLE, human neutrophil elastase; PA, pulmonary artery; PPE, porcine pancreatic elastase.

J. Clin. Invest.

(c) The American Society for Clinical Investigation, Inc. 0021-9738/94/09/1163/09 \$2.00

Volume 94, September 1994, 1163-1171 serious complication of congenital heart defects, pulmonary disease associated with chronic hypoxia, hepatic disorders, especially with portal hypertension, and connective tissue diseases. Pulmonary hypertension can also occur as a rapidly progressive disease of unknown etiology, which is usually fatal if not treated by lung transplantation (for review see reference 1 ). The vascular abnormalities associated with progressive pulmonary hypertension are related to structural and functional alterations in endothelial cells $(2,3)$ and changes in the smooth muscle cell phenotype. There is abnormal muscularization of normally nonmuscular peripheral arteries as a result of differentiation of precursor cells to smooth muscle (4), medial hypertrophy of more proximal muscular arteries associated with hypertrophy and hyperplasia of smooth muscle cells, and an increase in extracellular matrix, especially the connective tissue proteins, collagen and elastin. With further progression of pulmonary hypertension, there is obliterative intimal proliferation related to migration of smooth muscle cells into the subendothelium accompanied by an increase in the production of extracellular matrix components.

Previous studies by our group first showed through analysis of the ultrastructure of PA on lung biopsy from patients with congenital heart defects and pulmonary hypertension, that the internal elastic lamina, which normally separates endothelial from smooth muscle cells in muscular arteries, is fragmented (2). This suggested that an enzyme that has elastolytic properties might be involved in the pathophysiology of this poorly understood process. This was further explored in experimental rats in which progressive pulmonary hypertension was induced by injection of the toxin monocrotaline (5-9). There was an increased number of breaks in the internal elastic lamina observed as early as $4 \mathrm{~d}$ after injection of the toxin associated with the initiation of structural changes (7). Moreover, with progression of pulmonary hypertension and vascular disease 24 d later, high turnover of elastin was suggested by an increase in elastin synthesis out of proportion to accumulation (5). Subsequently, we confirmed an early increase in elastolytic activity that precedes vascular changes and a later increase associated with progressive disease (8). The nature of the inhibitor profile suggested that one or more serine proteinases were involved. We also showed evidence of increased serine elastolytic activity in PA of rats after only $2 \mathrm{~d}$ of exposure to chronic hypobaric hypoxia (10). However, with the development of sustained pulmonary hypertension associated with structural changes, i.e., medial hypertrophy, there was no further rise in elastolytic activity (10). Similar findings were evident in infant rats injected with the toxin monocrotaline but in this group of animals, as well as in rats exposed to hypoxia, medial hypertrophy was largely reversible $(8,10)$. Thus, we concluded that although the initial increase in elastolytic activity might be associated with the initiation of pulmonary vascular changes, the latter 
elevation might reflect progressive and potentially irreversible disease.

A cause and effect relationship between increased activity of an endogenous vascular elastase (EVE) and the development and progression of vascular changes and pulmonary hypertension in experimental rats was suggested by further studies in which we administered elastase inhibitors to rats either orally or, more effectively, by miniosmotic pump. In rats injected with the toxin monocrotaline, a 2-wk infusion of SC-37698 (Searle Pharmaceuticals, Chicago, IL), a serine elastase inhibitor, prevented increased EVE activity, vascular changes, and pulmonary hypertension. Even when the infusion was delayed for 1 wk and given only for the last 2 wk of a 3-wk experimental period, there was significant reduction in the severity of pulmonary hypertension and in the extent of vascular changes, including muscularization of peripheral arteries, medial hypertrophy of muscular arteries, and intimal thickening. This suggested that both the early increase in EVE activity at $2 \mathrm{~d}$ and the later increase between 16 and $28 \mathrm{~d}$ contribute to the development of pulmonary vascular changes (7). Further studies established that administration of serine elastase inhibitors, SC-39026, as well as $\alpha_{1}$-proteinase inhibitor, reduced hypoxia-induced pulmonary hypertension in rats (10). Administration of $\alpha_{1}$-proteinase inhibitor also reduces monocrotaline-induced pulmonary hypertension (9). In all cases, administration of the elastase inhibitors was associated with a reduction in EVE measured in the PAs of treated rats.

Previous studies by Hornebeck et al. (11) showed that an elastase was produced by aortic smooth muscle cells and associated with atherosclerosis, but this enzymatic activity was not further characterized. We therefore developed a molecular strategy to determine the nature of the pulmonary artery EVE activity. We produced degenerate oligonucleotides to conserved amino acid regions associated with the active sites of two serine elastases, human neutrophil elastase (HLE) and porcine pancreatic elastase (PPE). A PCR was done with rat PA cDNA. The product hybridized to a $1.2-\mathrm{kb}$ mRNA from rat $\mathrm{PA}$ and the intensity of hybridization was increased threefold in RNA from rat PA $28 \mathrm{~d}$ after monocrotaline injection, a time point when EVE activity was increased. The PCR product was also used to screen a cDNA library and the sequences from positive clones were shown to be identical to rat adipsin. Pulmonary artery EVE was then purified by immunoaffinity chromatography using a three-step procedure. A $20-\mathrm{kD}$ band was resolved as an active enzyme on an elastin substrate gel and was immunoreactive with the adipsin antibody. Using an antibody raised to an adipsin peptide sequence, we localized EVE to the PA smooth muscle cells.

\section{Methods}

mRNA preparation and generation of a PCR product. Pulmonary arteries from eight rats (180-200 g, Sprague-Dawley; Charles River Breeding Laboratories, Inc., Wilmington, MA) were harvested $28 \mathrm{~d}$ after a single $60 \mathrm{mg} / \mathrm{kg}$ injection of monocrotaline (Transworld Chemicals, Rockville, MD) as previously described (5). To extract RNA, the PA were homogenized in $3 \mathrm{ml}$ RNA zol/100 mg tissue (12) (Cinna/Biotex, Friendswood, TX). The homogenate was mixed with chloroform and centrifuged at $12,000 \mathrm{~g}$ for $15 \mathrm{~min}\left(4^{\circ} \mathrm{C}\right)$. The aqueous phase was transferred to a fresh tube, mixed with equal volume of isopropanol, and stored at $-20^{\circ} \mathrm{C}$ for $45 \mathrm{~min}$. The samples were centrifuged at 12,000 $g$ for $15 \min \left(4^{\circ} \mathrm{C}\right)$ to obtain RNA pellets. Poly $(\mathrm{A})+$ RNA was purified on oligo(dT)-cellulose (Pharmacia, Baie D'Urfe, Quebec, Canada). cDNA was reverse transcribed and used as a template in PCR and as an insert to generate a cDNA library. Two degenerate oligonucleotides encoding homologous amino acid regions of HLE and PPE were used as primers in the PCR reaction: primer 1 is 5' AAYTTYGTNATGZXNGCNGCNCAYTGYGT 3'; based upon amino acid sequences NFVMSAAHCV at 38-47 amino acid in HLE and NWVMTAAHCV at 35-44 in PPE; and primer 2 is 3' CCICTRZXICCIZXIGGIRAICAIACR 5', based upon amino acid sequences GDSGSPLVC at 186-194 in HLE and GDSGGPLHC at 171-179 in PPE. The spanning distance between the primers referred to above is $\sim 500$ bp $\mathrm{Y}=\mathrm{T}$ or $\mathrm{C} ; \mathrm{R}$ $=\mathrm{A}$ or $\mathrm{G} ; \mathrm{Z}=\mathrm{T}$ or $\mathrm{A} ; \mathrm{X}=\mathrm{G}$ or $\mathrm{C} ; \mathrm{N}=\mathrm{A}, \mathrm{C}, \mathrm{G}$ or $\mathrm{T}$; and $\mathrm{I}=$ inosine.

The PCR was performed using GeneAmp DNA Amplification Reagent Kit (Perkin-Elmer Cetus, Norwalk, CT) with $0.2 \mathrm{ng}$ of template and $1 \mu \mathrm{g}$ of each primer in $30 \mu \mathrm{l}$ of total volume. A single PCR product, $\sim 500 \mathrm{bp}$, was observed on a $1.2 \%$ agarose gel and extracted by placing a DE-81 membrane in front of the band. The gel was then run for 5 min more to move the PCR product onto the membrane. The PCR product was eluted from the membrane with $75 \mathrm{mM}$ Tris, $\mathrm{pH} 7.6$, $1 \mathrm{mM}$ EDTA, $1 \mathrm{M} \mathrm{LiCl}$, and $20 \%$ ethanol and was amplified by repeat PCR.

Northern blot analyses. RNA was extracted from PA in control rats, and rats 2 and $28 \mathrm{~d}$ after injection of monocrotaline as described above Northern blotting was performed using standard techniques (13). Approximately $15 \mu \mathrm{g}$ RNA per lane was loaded on a $1 \%$ agarose gel, transferred to nylon filters (Hybond; Amersham Corp., Arlington Heights, IL) by capillary elution for $24 \mathrm{~h}$ and fixed by exposure to short-wave ultraviolet irradiation. Hybridization was carried out using the ${ }^{32} \mathrm{P}$-labeled PCR product. The 1.4-kb cDNA probe for glyceraldehyde-3-phosphate dehydrogenase, used as a "housekeeping" gene, was purchased from Telios Pharmaceuticals, Inc. (La Jolla, CA) and isolated from pBR322 by PstI digestion.

Screening a rat PA $c D N A$ library. The ${ }^{32} \mathrm{P}$-labeled $\mathrm{PCR}$ product was used to screen a rat PA cDNA library. The cDNA library was commercially prepared (Stratagene, Inc., La Jolla, CA) and recombined in UniZAP phage. Positive plaques were isolated after the third screen and recombinant cDNA was excised by $\mathrm{R} 408$ helper phage. cDNA sequencing was carried out by the Sanger method using a T7 sequencing kit (Pharmacia).

Genomic DNA sequencing and derived $\mathrm{NH}_{2}$-terminal amino acid sequence. It appeared, on the basis of the previously published mouse cDNA sequence, that we were lacking an additional 21-bp related to the methionine start site in the sequences derived from the positive clones in our cDNA library. The full length sequence for the rat adipsin cDNA was completed by sequencing a PCR product obtained from genomic rat DNA. Two 18-bp primers, one at $-257 \mathrm{bp}$ of the previously published mouse adipsin start codon (14) and the other at +880 bp downstream, were used in PCR with rat genomic DNA as a template and PCR products were sequenced using the cycle sequencing method (15) (Pharmacia)

Elastase assay. At each step in the immunoaffinity purification procedure described below, elastolytic activity was tested as previously described (8). PA homogenates from eight rats, $28 \mathrm{~d}$ after injection of monocrotaline, were extracted at $4^{\circ} \mathrm{C}$ with $0.5 \mathrm{M} \mathrm{Na}$ acetate buffer, $\mathrm{pH}$ 4.0. The extracts were subsequently pooled, dialyzed against distilled water at $4^{\circ} \mathrm{C}$ for $24 \mathrm{~h}$, and then lyophilized. Samples from the initial PA extracts, as well as the wash-through and eluate from the various immunoaffinity columns, were each added to $20 \mu \mathrm{l}$ of ${ }^{3} \mathrm{H}$-elastin (specific activity $2,126 \mathrm{cpm} / \mu \mathrm{g}$ elastin ) and to Tris $-\mathrm{HCl}$ assay buffer. This enzyme-substrate mixture was incubated at $37^{\circ} \mathrm{C}$ for $18 \mathrm{~h}$ and the radioactive solubilized reaction products were measured with a scintillation counter.

Preparation of a polyclonal antibody to recombinant adipsin. To produce the adipsin antibody, we modified the procedure previously described (16). $100 \mu \mathrm{g}$ of mouse recombinant adipsin was dissolved in $750 \mu \mathrm{l}$ of PBS buffer and emulsified with an equal volume of complete Freund's adjuvant (Sigma Chemical Co., St. Louis, MO). The suspension $(1.5 \mathrm{ml})$ was injected at two sites into the pectoral muscle of a brown (Leghorn) laying hen, $20 \mathrm{wk}$ old. A further three injections of the protein, emulsified with the incomplete adjuvant, were given biweekly to 
the hen. The yolk $(\sim 10 \mathrm{ml})$, containing IgY antibodies, was purified from individual eggs after carefully separating it from the white, washing with deionized water, and then collecting it without the yolk skin. The yolk suspension was diluted in $30 \mathrm{ml}$ of $0.1 \mathrm{M}$ phosphate buffer ( $\mathrm{pH}$ 7.6) and mixed with a $10-\mathrm{ml}$ of solution of $0.175 \mathrm{~g} / \mathrm{ml}$ of polyethylene glycol (PEG) 8000 (Sigma Chemical Co.) dissolved in $0.1 \mathrm{M}$ phosphate buffer. The precipitate was pelleted by centrifugation at $5,000 \mathrm{~g}$ for 25 $\mathrm{min}$ at room temperature. The supernatant was filtered through filter paper. Solid PEG $(0.085 \mathrm{~g} / \mathrm{ml})$ was added to the supernatant and the mixture was stirred until all the PEG was dissolved. The solution was then centrifuged at $5,000 \mathrm{~g}$ for $25 \mathrm{~min}$ at room temperature. The pellet containing IgY was resuspended in $25 \mathrm{ml}$ of $0.1 \mathrm{M}$ phosphate buffer and mixed with $0.12 \mathrm{~g} / \mathrm{ml}$ of PEG for a second precipitation. The pellet was resuspended in $2.5 \mathrm{ml}$ of $0.1 \mathrm{M}$ phosphate buffer and an equal volume of $50 \%$ ethanol, left on ice for $\sim 4 \mathrm{~h}$, and then centrifuged at $10,000 \mathrm{~g}$ for $25 \mathrm{~min}$ at $4^{\circ} \mathrm{C}$. The pellet was dissolved in $2.5 \mathrm{ml}$ of the buffer and dialyzed against the same buffer and then against $0.01 \mathrm{M}$ phosphate buffer and $0.15 \mathrm{M} \mathrm{NaCl}, \mathrm{pH}$ 7.6.

Immunoaffinity purification of EVE. The PA sample, extracted as described above, was applied to three successive affinity columns. The three antibodies were conjugated to $0.3 \mathrm{ml}$ affigel (Bio-Rad Laboratories, Inc., Richmond, CA) with $0.1 \mathrm{M}$ bicarbonate buffer, $\mathrm{pH} 7.75$, which was also used in three purification procedures. In the first column, the antibody (BCZ) recognizes the lectin site of the elastin binding protein (EBP) (17) and was kindly provided by Dr. Robert Mecham of Washington University (St. Louis, MO). The PA extract was incubated with the $\mathrm{BCZ}$ for $1 \mathrm{~h}$. In the second column, the anti-S-GAL antibody recognizes both the EBP (removed by the first column) and the $\mathrm{NH}_{2}$-terminal regions of serine elastases (18). The wash-through from BCZ column was then applied to the anti-S-GAL column for 1 $\mathrm{h}$ and eluted with $8 \mathrm{M}$ urea. The eluate was then applied to a third column, which was conjugated with an antibody to recombinant mouse adipsin for $1 \mathrm{~h}$ and eluted with $8 \mathrm{M}$ urea.

$S D S$-substrate gel for detecting EVE. An SDS-substrate gel was used to localize the PA-EVE activity by molecular weight (19). The gel was made by incorporating $0.67 \mathrm{mg} / \mathrm{ml}$ of soluble elastin substrate (Elastin Products, Pacific, MO) within $10 \%$ polymerized acrylamide matrix and the PA samples were mixed with $10 \%$ SDS buffer without reducing agents or heating. The gel was run at $4^{\circ} \mathrm{C}$ to reduce enzyme interaction with the substrate and at $15 \mathrm{~mA} / \mathrm{gel}$ while stacking and 20 $\mathrm{mA} /$ gel during the resolving phase. The proteins were allowed to renature by removing the SDS (which was accomplished by soaking in $2.5 \%$ Triton X-100 with gentle shaking for $30 \mathrm{~min}$ at ambient temperature with one change). The gel was then rinsed in substrate buffer and incubated in substrate buffer (Tris- $\mathrm{HCl}$ ) at $37^{\circ} \mathrm{C}$ for $5 \mathrm{~d}$ with gentle shaking. At the end of the incubation, the gel was stained with Coomassie blue for 30-60 min with shaking and destained in water. The area containing enzyme was pale against a blue background.

Western immunoblot. To confirm whether the elastolytic band on the substrate gel was immunoreactive with the adipsin antibody, Western immunoblots were carried out. The samples were suspended in SDS sample buffer and the proteins were resolved by using $8-16 \%$ gradient Tris-glycine "Laemmli" precast PAGE gel and then transferred to a polyvinylidene difluoride (PVDF) membrane at $30 \mathrm{~V}$ for $1.5 \mathrm{~h}$ (NOVEX, San Diego, CA). The membrane was then immunoblotted with the antibody to recombinant mouse adipsin (produced as described above) diluted 1:300. The reaction was visualized using the alkaline phosphatase-conjugated rabbit anti-chicken IgG antibody (Sigma Chemical Co.) diluted 1:300. 5-bromo-4-chloro-3-indolylphosphate $p$-toluidine salt and nitroblue tetrazolium chloride (Gibco BRL, Grand Island, NY) (1:1 molar ratio) were used as chromagenic substrates for the enzyme alkaline phosphatase and resulted in a blue color reaction to positive proteins.

Localization of elastase in PA tissue. A polyclonal antibody was raised in a rabbit (by Dr. B. Starcher, Tyler, TX) to a peptide sequence from adipsin (see Fig. 2, the underlined sequence screened in the gene bank and not found to be present in other proteins) and used to localize PA-EVE by immunoelectron microscopy. We followed the procedure previously described $(20)$ in which rat PA sections were fixed in $0.5 \%$

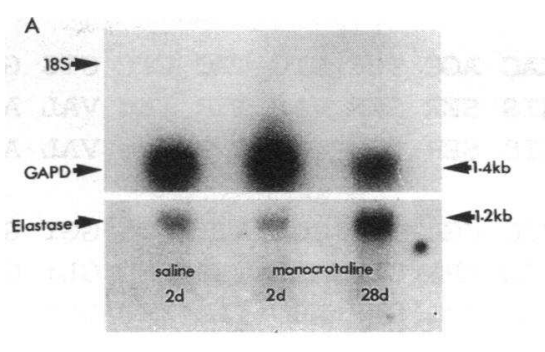

B

* $p<0.001$

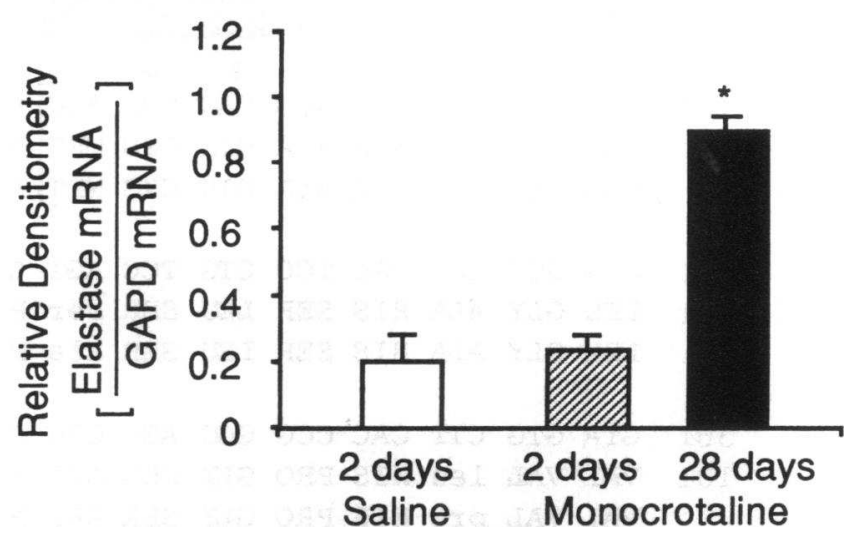

Figure 1. Northern blot analysis of putative PA endogenous vascular elastase mRNA levels at different time points after saline or monocrotaline injection. $(A)$ A representative Northern blot shows that the ${ }^{32} \mathrm{P}$ labeled PCR product (putative elastase $\mathrm{CDNA}$ ) hybridizes to a single mRNA, $1.2 \mathrm{~kb}$; glyceraldehyde-3-phosphate dehydrogenase (GAPD) used as a "housekeeping" gene is seen as a 1.4-kb band. (B) A graph shows densitometric data from four different experiments. No increase in intensity of hybridization with RNA was shown in rats $2 \mathrm{~d}$ after monocrotaline compared with saline injection, whereas a threefold increase in intensity of hybridization signal was observed comparing PA RNA from rats $28 \mathrm{~d}$ after injection of monocrotaline with saline (control) rat PA RNA $(P<0.001$, ANOVA, and Duncan's multiple range test).

glutaraldehyde and $0.5 \%$ paraformaldehyde in $0.1 \mathrm{M}$ Tris-buffered saline, pH 7.4. Reactive aldehydes were blocked with $0.5 \mathrm{M}$ glycine, the samples were washed with Tris-buffered saline, postfixed with $1 \%$ osmium tetroxide in $0.1 \mathrm{M}$ cacodylate buffer, dehydrated in ethanol, and embedded in Epon. The sections were placed on nickel grids blocked with bovine serum albumin, $3 \%$ normal goat serum, and $0.5 \%$ Tween 20 in Tris-buffered saline and reacted with the adipsin peptide polyclonal antibody. The immune reaction was visualized with secondary antibody (goat anti-rabbit conjugated with $15 \mathrm{~nm}$ gold particles). Sections were then stained with uranyl acetate and lead citrate and visualized using an electron microscope (Philips 201; Philips Electronic Instruments, Mount Vernon, NY).

\section{Results}

PCR product and Northern blot analysis. The PCR produced a single product of $\sim 500 \mathrm{bp}$, which was further amplified and eluted from the gel. This product was the approximate spanning distance between the degenerate oligonucleotides chosen, which encode for cDNA sequences of HLE and PPE. When used in Northern blots loaded with total RNA extracted from rat PA, the radiolabeled PCR product hybridized to a single 1.2-kb mRNA (Fig. 1). No increase in intensity of hybridization signal 
Rat [ CDNA ATg CAC ACC TCC GTG IAC CTC GTG GCT CTG GTG GTC CTG GAG GCG GCT GTA TGi GTT GCG

AA MET HIS SER SER VAL TYR Ieu VAL AIA LEU VAL val LEU GLU ALA ALA VAL CYS Val ALA Mouse AA MET HIS SER SER VAL TYR phe VAL ALA LEU VAL Ile LEU GLY AIA ALA VAL CYS ala ALA

61 CAG CCC CGA GGT CGG ATT CTG GGT GGC CAG GAG GCC ATG GCC CAT GCT CGG COC TAC ATG 21 GLN PRO ARG GLY ARG IIE LEU GLY GLY GLN GLU AIA met ALA HIS ALA ARG TRO TYR MET GLN PRO ARG GLY ARG IIE LEU GLY GLY GLN GLU AIA ala ALA HIS ALA ARG TRO TYR MET

121 GCT TCA GTG CAA GTG AAT GGC ACG CAC GTG TGC GGT GGC ACC CTG GTG GAT GAG[CAG TGG

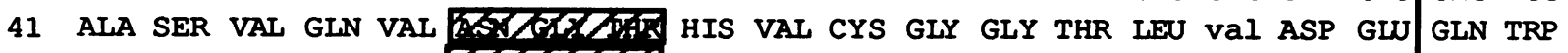

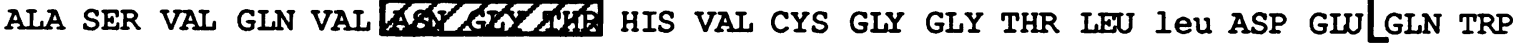

$$
70
$$

181 GTG CTG AGC GCC GCG CAC TGC ATG GAT GGA GTG ACC AAG GAT GAG GTT GTG CAG GTG CTC

61 VAL LEU SER ALA ALA HIS CYS MET ASP GLY VAL THR IYS ASP glu Val VAL GIN VAL LEU VAL LEU SER ALA ALA HIS CYS MET ASP GLY VAL THR asp ASP asp ser VAL GIN VAL LEU

241 CTG GGT GCC CAC TCC CTG TCC AGT CCT GAA CCC TAC AAG CAT TTG TAT GAT GTG CAA AGT

81 LEU GLY AIA HIS SER LEU SER SEY PRO GLU PRO TYR LYS his leu TYR ASP VAL GLN SER LEU GLY AIA HIS SER LEU SER ala PRO GLU PRO TYR LYS arg trY TYR ASP VAL GLN SER

301 GTA GTG CTT CAC CCG GGC AGC CGG CCT GAC AGC GTT GAG GAC GAC CTC ATG CTC TTT AAG

101 VAL VAL leu HIS PRO GLY SER ARG PRO ASP SER val GLU ASP ASP LEU met LEU PHE LYS VAL VAL prO HIS PRO GLY SER ARG PRO ASP SER leu GLU ASP ASP LEU ile LEU PHE LYS

361 CTC TCC CAC AAT GCC TCA CTG GGT CCC CAT GTG AGA CCC CTG CCC TTG CAA CGC GAG GAC

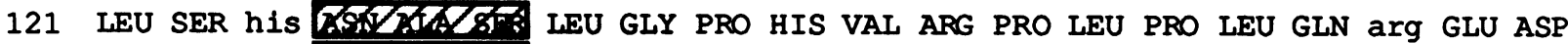

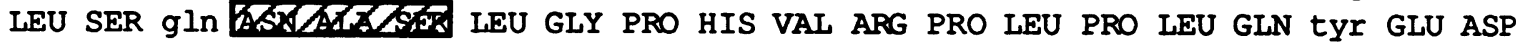

421 CGG GAG GTG AAA CCC GGC ACG CTC TGC GAT GTG GCC GGT TGG GGC GTG GTC ACT CAT GCG 141 arg GLU VAL lYS PRO GLY THR LEU CYS ASP VAL ALA GLY TRP GLY VAL VAL THR HIS ALA lYS GLU VAL glu PRO GLY THR LEU CYS ASP VAL AIA GLY TRP GLY VAL VAL THR HIS ALA

481 GGA CGC AGG CCC GAT GTC CTG CAG CAA CTG ACA GTG TCA ATC ATG GAC CGG AAC ACC TGC 161 GLY ARG ARG PRO ASP VAL LEU gln GLN LEU thr VAL SER ILE MET asp ARG asn THR CYS

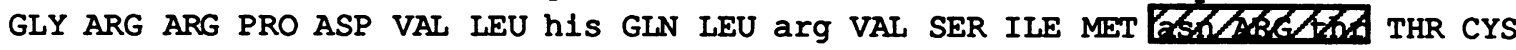

541 AAT CTG CGC ACG TAC CAT GAT GGg GCA ATC ACC AAg AAC ATG ATG TGT GCA GAg AGC AAC 181 ASN LEU ARG THR TYR HIS ASP GLY ala 1le THR lyS ASN MET MET CYS ALA GL SER ASN ASN LEU ARG THR TYR HIS ASP GLY val val THR 1le ASN MET MET CYS ALA GLU SER ASN

601 CGC AGG GAC ACT TGC AGG[GGC GAC TCC GGC GGT CCT CTG GTG TGC]GGG GAT GCG GTC GAA 201 ARG ARG ASP THR CYS ARG GLY ASP SER GLY GIY PRO LEU VAL CYS GLY ASP AIA VAL GLU ARG ARG ASP THR CYS ARG GLY ASP SER GLY ser PRO LEU VAL CYS GLY ASP ALA VAL GLU -

661 GCT GTG GTT ACG TGG GGA TCT CGA GTC TGT GGC AAC CGG AGA AAG CCA GGT GTC TTT ACC 221 ala VAL VAL THR TRP GLY SER ARG VAL CYS GLY ASN arg arg LYS PRO GLY VAL phe THR glY VAL VAL THR TRP GLY SER ARG VAL CYS GLY ASN glY lYS LYS PRO GLY VAL TYY THR

721 CGC GTG GCA ACC TAC GTG CCG TGG ATT GAA AAC GTT CTG AGT GGT AAC GTG AGT GTT AAC

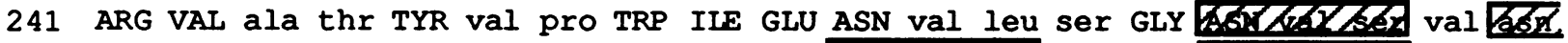

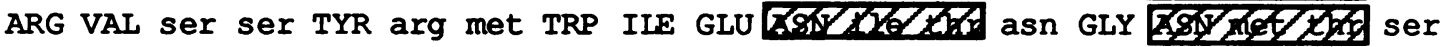

781 GTG ACG GCC TGA GGGGACACCGGAGACCGTGACTCACAATAAATGCATGCATCTAAAAAAAAAAAA

261 arepara ala stop 
Table I. Relative Endogenous Vascular Elastase Activity during the Purification Procedure

\begin{tabular}{lcc}
\hline & Degraded ${ }^{3}$ H-elastin & $\begin{array}{c}\text { Percent elastase activity/total } \\
\text { (wash + eluate) }\end{array}$ \\
\hline $\begin{array}{c}\text { Initial PA extract } \\
\text { Wash }\end{array}$ & 71 & \\
BCZ & 14,370 & 99.7 \\
$\quad$ Eluate & 14 & - \\
$\quad$ Wash & 2,139 & - \\
$\begin{array}{l}\text { Anti-S-GAL } \\
\text { Eluate } \\
\text { Wash }\end{array}$ & 68,692 & 97.0 \\
Anti-adipsin & 5,814 & - \\
Eluate & 60,601 & 91.2 \\
\end{tabular}

The elastase activity in the initial PA extract during a 5-hr incubation was $71 \mathrm{cpm}$ degraded ${ }^{3} \mathrm{H}$-elastin/10 mg PA tissue, which is equivalent to $2.5 \mathrm{ng}$ of HLE activity. After BCZ immunoaffinity purification, there was a $>100$-fold increase in elastolytic activity detected in the wash with $<1 \%$ retrieved from the eluate. A further nearly fivefold increase in elastolytic activity was detected in the eluate after anti-S-GAL immunoaffinity purification. Only $3 \%$ of the total activity was present in the wash. When the anti-S-GAL eluate was applied to the antiadipsin immunoaffinity column, $<10 \%$ of the activity was detected in the wash.

was evident with RNA from rats $2 \mathrm{~d}$ after injection, whereas a threefold increase in intensity of hybridization signal was evident when comparing PA RNA of rats $28 \mathrm{~d}$ after injection of monocrotaline with control rat PA RNA $(P<0.001$, ANOVA, and Duncan's multiple range test). The 1.2-kb mRNA to which our PCR probe hybridizes is similar in size to the adipsin mRNA previously described (21).

cDNA and genomic DNA sequencing. Three different positive overlapping clones were found upon screening of the cDNA library. Sequencing of these clones generated $\sim 800 \mathrm{bp}$, which were $90 \%$ homologous to mouse adipsin (Fig. 2) (14). Rat adipsin had been independently sequenced by Drs. Bruce Speigelman (Dana Farber Cancer Institute, Harvard Medical School, Boston, MA) and Tyler White (Scios, Nova Inc., Mountain View, CA), who kindly shared their sequence data with us. The structures we derived were $100 \%$ identical to theirs. Neither these investigators nor our group had been successful in cDNA sequencing of the rat adipsin start codon region, the first 21 bases (seven amino acids), as judged by the mouse sequence. We therefore established this sequence from genomic DNA using cycle sequencing of PCR products (Fig. 2, bold). The rat sequence in this region differed from the mouse sequence by one amino acid. With respect to the rat adipsin cDNA sequence, the regions corresponding to the oligonucleotides used in PCR were present and are indicated by the brackets on Fig. 2. In addition, potential glycosylation sites, as seen in the

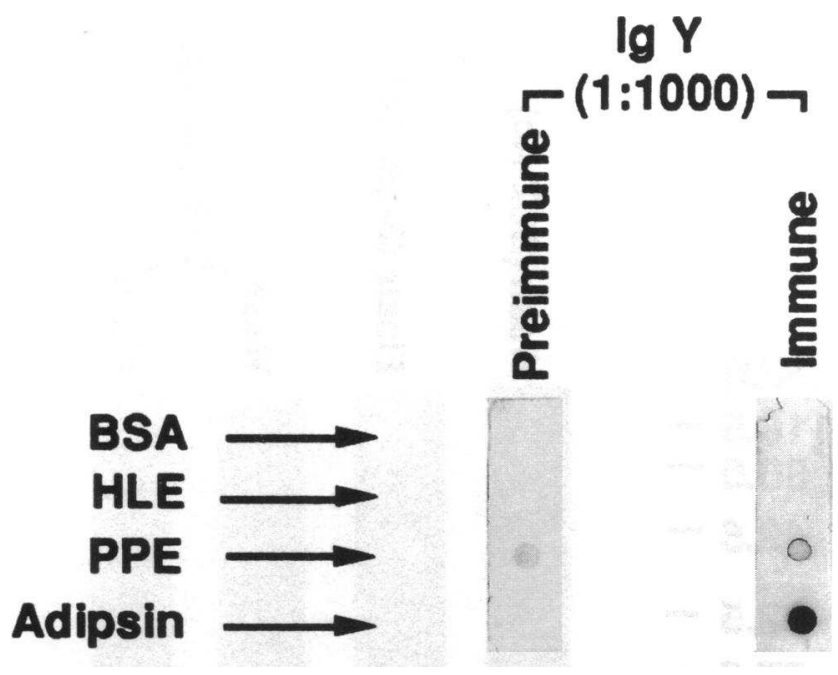

Figure 3. Dot blot analysis verifying the specificity of antiadipsin antibody. A dot blot shows that the antibody prepared in egg yolk (IgY) and used in the affinity purification and Western immunoblot reacts positively at a titre of 1:1,000 with recombinant adipsin but not with human leukocyte elastase $(H L E)$, porcine pancreatic elastase $(P P E)$, or bovine serum albumin $(B S A)$. Preimmune $\operatorname{IgY}$ is not reactive with these elastases or with adipsin or with BSA.

mouse sequence, are present and indicated by the hatched bars. The conserved cysteines and the three key amino acids, related to the active site of the enzyme: histidine 66, aspartic acid 114 , and serine 209, are all present at the appropriate locations (Fig. 2, arrow heads).

Immunopurification of EVE with an antibody to adipsin. In other studies in our laboratory, we identified a sequence, $S$ GAL, from the alternatively spliced variant of $\beta$-galactosidase with homology to the $\mathrm{NH}_{2}$ terminal of serine elastases $(18)$ and to the $67-\mathrm{kD}$ EBP $(17,18)$. The EBP appears to compete with elastases for elastin and thereby serves as an inhibitor of elastolytic activity (22).

We therefore used this information, specifically the homology between serine elastases and EBP, to purify EVE from rat PA after injection of monocrotaline. The rat PA homogenates were prepared and an aliquot taken for assessment of elastolytic activity using a ${ }^{3} \mathrm{H}$-elastin substrate as previously described (8). The sample was then applied to three successive affinity columns. In the first column, affigel was bound to $\mathrm{BCZ}$ antibody, which recognizes the lectin site of EBP. The removal of EBP enhanced the elastolytic activity over 100-fold in the wash compared with the initial extract, whereas there was no elastolytic activity present in the eluate (Table I). The wash-through was then applied to an anti-S-GAL antibody column, i.e., the antibody that recognizes both the EBP (removed by the first col-

Figure 2. The putative cDNA sequence of EVE obtained from overlapping positive clones from a rat PA cDNA library. The sequences 813 bp upstream from the poly (A) + tail are $100 \%$ homologous with the cDNA sequence corresponding to the coding region of rat adipsin, a serine elastase not previously reported in vascular or lung tissue but sequenced independently by Drs. Bruce Spiegelman and Tyler White as described in Methods. On the basis of the published amino acid $(A A)$ sequence of mouse adipsin, which is $90 \%$ homologous with rat adipsin, sequences derived from the cDNA library begin 21 bases from the start codon. Indicated in bold is the rat adipsin 21 bp and seven amino acid sequences derived from genomic DNA as described in Methods. Differences in the mouse sequence are illustrated in lowercase type. The regions corresponding to the oligonucleotides used in PCR are bracketed, the potential glycosylation sites are indicated by the hatched bars, the conserved cysteines are indicated by dots, and the three key amino acids related to the active site of the enzyme are indicated by arrowheads. We have underlined the sequence used in preparing the polyclonal antibody used to immunolocalize adipsin in the PA. 


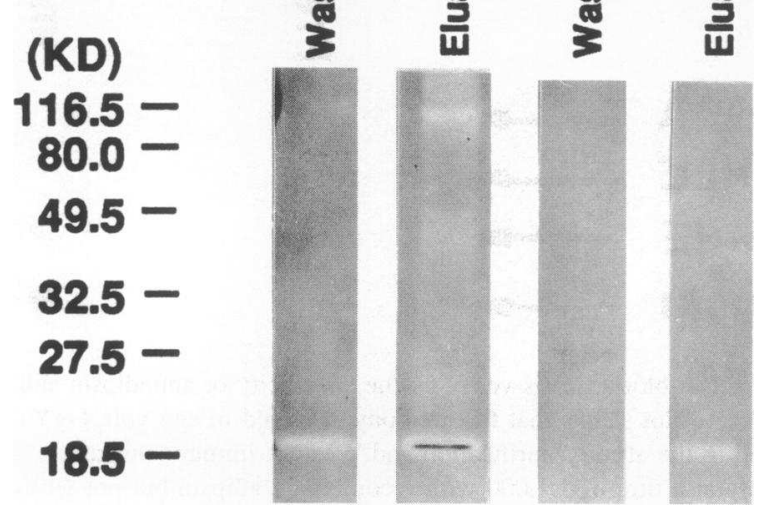

Figure 4. An elastin substrate gel to resolve the molecular weight of the PA elastase enzyme. A substrate gel with elastin $(0.67 \mathrm{mg} / \mathrm{ml})$ impregnated in $10 \%$ polyacrylamide shows that in the wash after the BCZ (antielastin binding protein) immunoaffinity column, the eluate after the anti-S-GAL antibody (antibody that recognizes $\mathrm{NH}_{2}$-terminal sequences of elastase) immunoaffinity column, and the eluate after the antiadipsin antibody immunoaffinity column, but not the wash, contain an elastase enzyme (the lytic band at $20 \mathrm{kD}$ ). A band at $\sim 100 \mathrm{kD}$ in the S-GAL eluate may represent elastase bound to $72-\mathrm{kD}$ elastin, but it was not seen in the wash and did not bind to the antiadipsin antibody immunoaffinity column.

umn ) and the $\mathrm{NH}_{2}$-terminal regions of serine elastases $(18,22)$. There was minimal elastase activity in the second wash, as $97 \%$ adhered and could be recovered from the eluate of this second column. The eluate was then applied to a third column, which was conjugated with an antibody to recombinant mouse adipsin produced in a chicken. Over $90 \%$ of the elastolytic activity present in the eluate from the second column bound to the antiadipsin antibody affinity column and could subsequently be eluted.

The specificity of the antiadipsin antibody was evident in that there was no cross-reactivity with HLE or PPE (Fig. 3). Fig. 4 shows an elastin substrate gel reflecting the EVE activity through this purification procedure. Fig. 5 shows a Western immunoblot confirming that the elastolytic band on the substrate gel (20-kD protein) was immunoreactive with the adipsin antibody, which recognizes recombinant mouse adipsin (44- and 36-kD proteins) but not HLE and PPE. The product of translation of adipsin mRNA is $\sim 28 \mathrm{kD}$, including a propeptide region, but mouse adipsin is heavily glycosylated and, on Western immunoblot, the posttranslational product is recognized as two closely related proteins of 36 and $44 \mathrm{kD}$ of molecular mass (21). Thus, EVE was recognized and purified by antibodies to the $\mathrm{NH}_{2}$ terminal of elastases, as well as to an antibody prepared to recombinant mouse adipsin, suggesting, together with the sequence data, that the EVE associated with experimental pulmonary hypertension is related to adipsin.

Immunolocalization of EVE. We next showed that the smooth muscle cells in PA are the major source of EVE in the

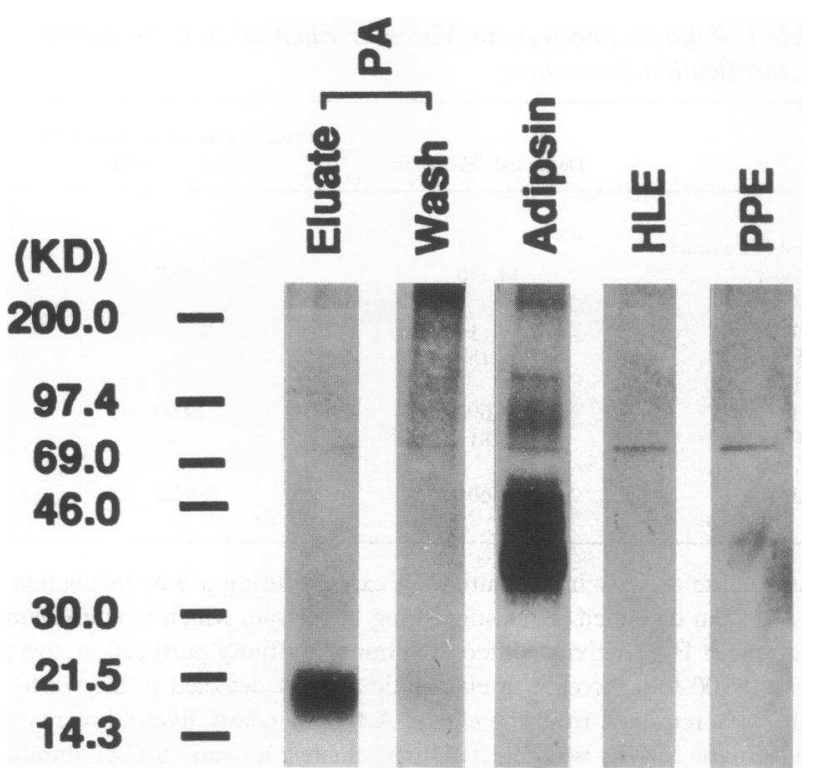

Figure 5. Western immunoblot of pulmonary artery $(P A)$ elastase using antiadipsin antibody. Western immunoblot showing that from the PA extract, a $20-\mathrm{kD}$ protein purified using the immunoaffinity procedure described in Methods and in Fig. 4 is immunoreactive with the antiadipsin antibody (1:300), as is recombinant adipsin but not human leukocyte $(H L E)$ or porcine pancreatic elastases $(P P E)$. The PA eluate denotes the material eluted from the antiadipsin immunoaffinity column, the final stage in the purification procedure.

vessel wall. A polyclonal antibody was prepared to the adipsin peptide sequence: Cys Ala Glu Ser Asn Arg Arg Asp. Immunogold localization showed that most of the reactive sites were in the secretory apparatus of smooth muscle cells and some were localized at the cell surface and in the extracellular matrix closely associated with elastin (Fig. 6).

\section{Discussion}

We have provided cDNA sequence and immunologic evidence relating adipsin to a novel enzyme, the EVE associated with the development and progression of pulmonary hypertension. Endogenous vasculsar elastase appears to be processed and secreted by the PA smooth muscle cells of the arterial wall and may be related to the serine proteinase previously described (11) but hitherto uncharacterized in aortic smooth muscle cells and in atherosclerosis.

Neither recombinant nor adipocyte or adipose tissue-derived adipsin appears to have elastolytic activity (unpublished observations; we have carried out assays with recombinant adipsin, adipose tissue, and supernatants from adipocytes [the latter kindly supplied by Dr. Amira Klip, Hospital for Sick Children, Toronto, Ontario, Canada] using the procedure described in this paper and have been unable to document elastolytic activity). Adipsin is a secreted protein found in the serum and first identified in mouse adipose tissue and in sciatic nerve and is $30 \%$ homologous to trypsin, chymotrypsin, and elastases (23) (Fig. 7 ). Although rat adipsin is the homologue of human complement factor D and cleaves factor B (24), its presence in fat tissue suggested that it has other functions related to fat metabolism. In fact, adipsin is upregulated in starvation and downregulated with obesity (21). It is of interest that complement factor 

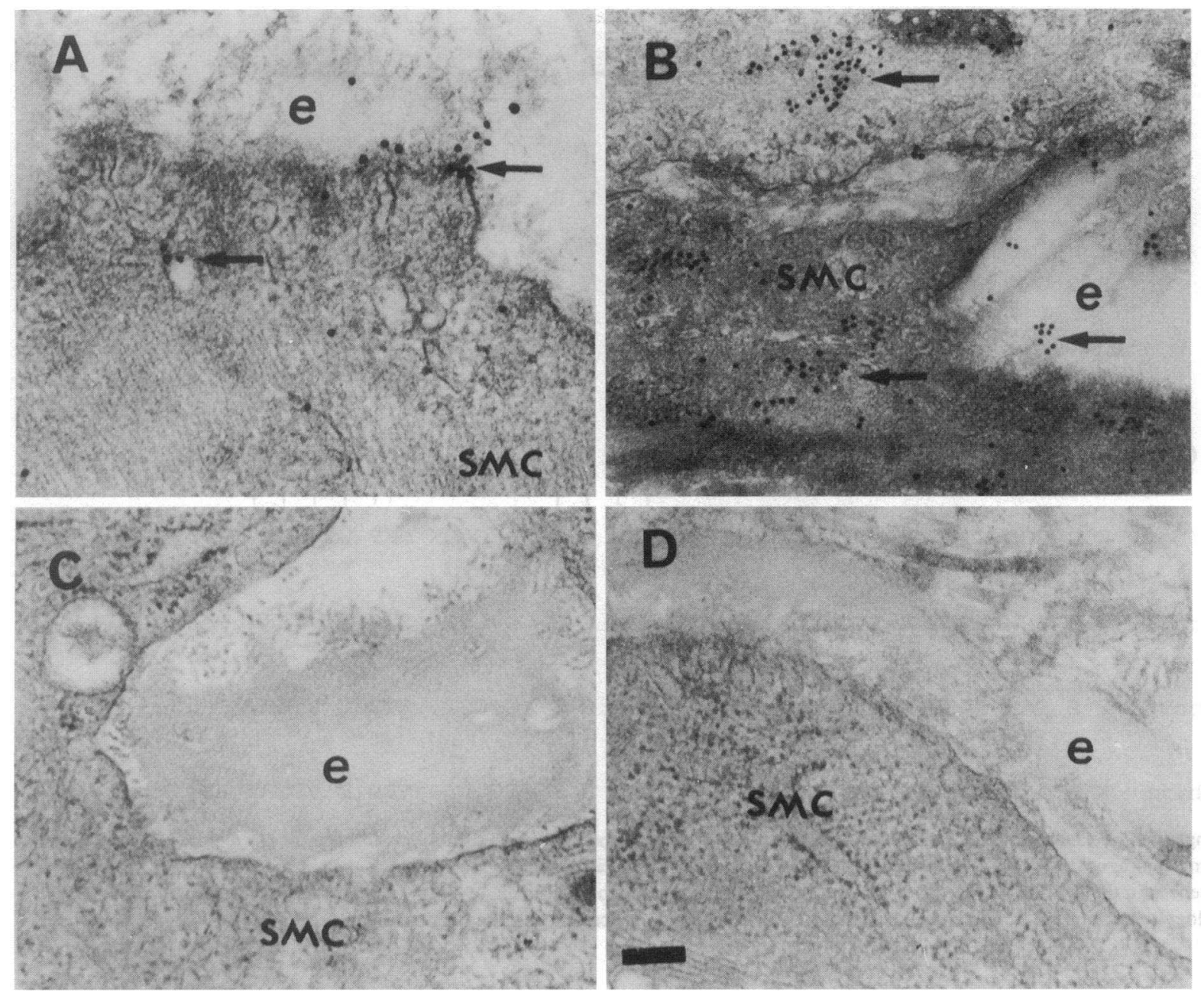

Figure 6. Representative immunoelectron photomicrographs using an antiadipsin peptide antibody. An antibody was raised in a rabbit to the adipsin sequence: Cys Ala Glu Ser Asn Arg Arg Asp. Goat anti-rabbit antibody was conjugated with 15-nm gold particles and hybridized to the antiadipsin antibody reflecting the antigenic sites (arrows). (A) A smooth muscle cell $(S M C)$ from a rat pulmonary artery $28 \mathrm{~d}$ after injection of monocrotaline shows adipsin antigenic sites related to the cell surface, secretory vesicles, and in close proximity with elastin $(e)$. $(B)$ The antibody is seen more extensively over the SMC and seems to be associated with the Golgi apparatus. In $C$, the antibody has been preabsorbed to an adipsin affinity column and no or only rare antigenic sites were apparent on the tissue. In $D$, the tissue was immunoreacted with normal rabbit serum and there are no positive sites. Magnification 48,600. Bar, $100 \mathrm{~nm}$.

C3a has recently been shown to be responsible for fat acylation (25). Although adipsin has not been shown to have proteolytic functions other than those related to cleavage of factor $B$, it has structural features typical of a serine proteinase and is homologous to other serine proteinases as well as elastases, particularly in the region of the active site of these enzymes (Fig. 7).

Our studies suggest several reasons why adipsin and EVE are related. The degenerate oligonucleotides should have picked out the cDNA for any serine elastase expressed in the PA tissue, but there was only one PCR product, and it only hybridized to clones in the PA cDNA library that encoded adipsin. Furthermore, the antibody that recognizes the $\mathrm{NH}_{2}$ terminal of serine proteinases should have selected out all serine proteinases, including adipsin if it was present in the tissue extract. The only protein in the PA extract that cross-reacted with the antibody to adipsin was, however, $20 \mathrm{kD}$, i.e., the molecular mass of the elastolytic enzyme. Finally, an antibody raised to recombinant adipsin isolated EVE from the PA extract.

How then might adipsin be related to EVE? It is possible that EVE is a product of posttranslational modification of adip- sin. This would suggest that some modification is necessary to elicit elastolytic activity. We have tried to convert recombinant adipsin to elastase by deglycosylation and by incubating with other serine proteinases, such as plasmin or thrombin, and detergents, namely, DTT and DMSO, or with the toxin monocrotaline. We also incubated adipsin with PA extracts to determine whether some factor in the tissue would be necessary for its activation to an elastolytic enzyme. None of these approaches resulted in elastolytic activity. We have also tried to mimic the lysosomal environment by acidification, but this resulted in minimal increases in elastolytic activity.

A recent study has shown that actin isoforms that differ at the $3^{\prime}$ UTR distribute differently within the cell (26). Although it is theoretically possible that there are adipsin isoforms that differ at the $3^{\prime}$ UTR and that one of these isoforms traffics to a cellular compartment in which it is processed to EVE, both rat adipsin and the PA cDNA sequences that we obtained are similar at the $\mathrm{COOH}$ terminal. It is also possible that there may be different $\mathrm{NH}_{2}$-terminal sequences for EVE and adipsin that result in different processing and/or functional properties. We 


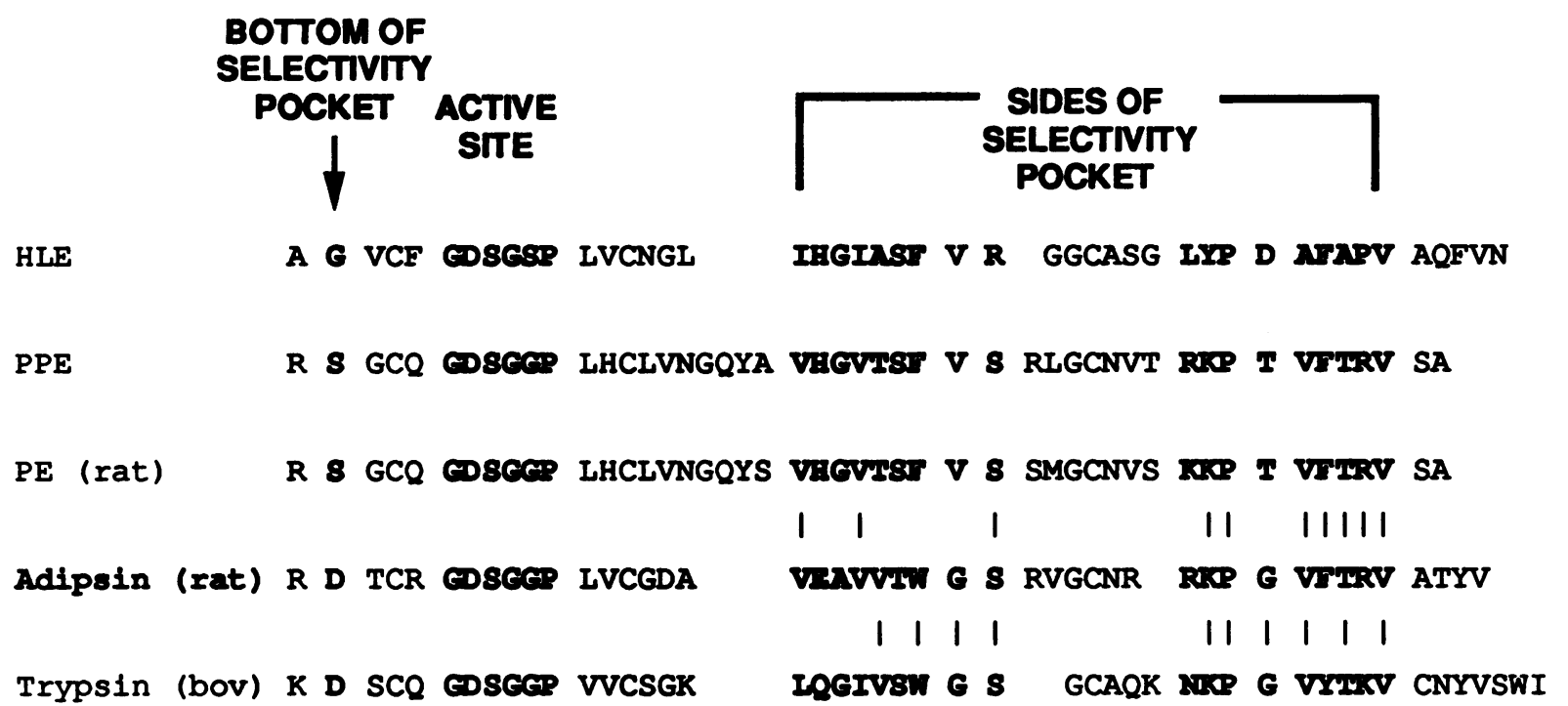

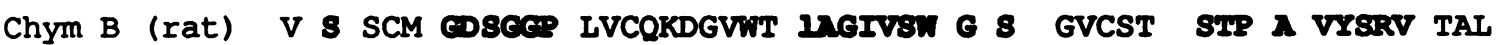

Chym A (bov) V 8 SCM CDSGeP IVCKKNGANT IVGIVEW G 8 STCST SIP G VIERV TAL

Cath G (hum) K I AFK GDSGCP ILCNN VAHGIVSY G K SSG VPR E VIIRV SS

Figure 7. Comparison of the structure of rat adipsin with that of other serine proteinases. Adipsin seems to have all the requirements for trypsinlike activity: an aspartic acid $(D)$ at the bottom of the selectivity pocket and small residues (glu or ala) at the sides of the pocket. On the other hand, the sequences in the area of the selectivity pocket are more homologous to rat pancreatic elastase than to trypsin or chymotrypsin. Bars indicate homology to adipsin. HLE, human leukocyte elastase; PPE, porcine pancreatic elastase; PE, pancreatic elastase; bov, bovine; hum, human.

established the $\mathrm{NH}_{2}$-terminal sequence reported in this paper from rat genomic DNA by PCR using a mouse-derived adipsin primer. It is therefore possible that the cDNA from the PA has a different $\mathrm{NH}_{2}$-terminal sequence. Although EVE could be derived from an alternatively spliced mRNA, there is no evidence as yet to support alternative splicing of adipsin mRNA (14). An alternative hypothesis is that EVE and adipsin share common coding region sequences but are derived from different genes.

It is interesting that there was increased intensity of hybridization to rat PA RNA harvested $28 \mathrm{~d}$ after injection of monocrotaline using the cDNA derived from the PCR reaction, which suggests that EVE mRNA is increased at this time point and this is consistent with the enzymatic activity previously described (8). The lack of increase at $2 \mathrm{~d}$ after monocrotaline, despite the increase in enzymatic activity, may reflect posttranscriptional regulation or release or activation of enzyme from storage sites.

EVE may play an important role in vascular remodeling in both the embryo and in the postnatal development of the vasculature. Using the antiadipsin antibody, we have seen expression of EVE in embryonic (E-18.5) lung tissue in association with larger preacinar arterial development as well as angiogenesis (unpublished observations; Studies currently in progress with Drs. Clayton Buck and Jon Edelman, Wistar Institute, Philadelphia, PA.). It is likely that, as vessels grow in diameter, the increase in diameter of the elastic lamina would require a remodeling mechanism involving EVE activity and elastin synthesis. Elastin peptides may, in fact, stimulate elastin synthe- sis by a feedback mechanism as has been suggested in other tissues (27).

Our previous studies suggest that activation of elastase in disease may be related to endothelial injury (2) and penetration of a serum factor (28). The serum factor appears to increase the affinity of elastin for the EBP on the cell surfaces and this in turn induces elastase activity via a tyrosine kinase mechanism. Since serum-induced EVE activity is not only seen with PA cells but also with systemic arterial cells, it is possible that the increased activity of this enzyme plays a role in a variety of vasculopathies. We have shown increased activity of a serine elastase of similar molecular weight in coronary arteries of piglets after experimental induction of the postcardiac transplant arteriopathy (29). Increased activity of a serine elastase is also present in aorta organ culture in association with the development of neointimal formation (30). In the clinical setting, increased elastolytic activity has also been described in atherosclerosis (11) and in a vasculopathy induced by actinic radiation (31).

The mechanism whereby increased activity of EVE might lead to proliferation and migration of smooth muscle cells and increased production of extracellular matrix culminating in the neointimal lesion is not known. It is possible that, like other serine proteinases (32-34), EVE releases growth factors, such as basic fibroblast growth factor or transforming growth factor$\beta$, from the extracellular matrix and activates them. These growth factors $(35,36)$ among others $(37,38)$ have been implicated in pulmonary (35) and systemic vascular pathology (36).

Thus, we have for the first time isolated and characterized 
an EVE. This enzyme has been previously shown to play a key role in the pathophysiology of pulmonary hypertension (8) and may also be present in coronary arterial disease associated with neointimal formation (29). Endogenous vascular elastase appears to be a novel enzyme related to the serine proteinase adipsin, although the precise nature of that relationship still remains to be determined.

\section{Acknowledgments}

This work was supported by a grant (T-2229) from the Heart and Stroke Foundation of Ontario. H. Dodo was supported by a scholarship from the University of Tokyo, M. Zuker was supported by a Summer Studentship from the Department of Pathology, J. Kobayashi was supported by a Medical Research Council of Canada Fellowship, and A. Hinek and M. Rabinovitch are Career Investigators of the Heart and Stroke Foundation of Ontario.

\section{References}

1. Rabinovitch, M. 1989. Pulmonary hypertension. In Heart Disease in Infants, Children, and Adolescents. A. J. Moss, F. M. Adams, and G. C. Emmanouilides, editors. Williams and Wilkins, Baltimore. 856-885.

2. Rabinovitch, M., T. Bothwell, B. N. Hayakawa, W. G. Williams, G. A. Trusler, R. D. Rowe, P. M. Olley, and E. Cutz. 1986. Pulmonary arterial endothelial abnormalities in patients with congenital heart defects and pulmonary hypertension: a correlation of light with scanning electron microscopy and transmission electron microscopy. Lab. Invest. 55:632-653.

3. Rosenberg, H. C., and M. Rabinovitch. 1988. Endothelial injury and vascular reactivity in monocrotaline pulmonary hypertension. Am. J. Physiol. 255:H1484-H1491.

4. Meyrick, B., and L. Reid. 1980. Ultrastructural findings in lung biopsy material from children with congenital heart defects. Am. J. Pathol. 101:527537.

5. Todorovich-Hunter, L., D. J. Johnson, P. Ranger, F. W. Keeley, and M. Rabinovitch. 1988. Altered elastin and collagen synthesis associated with progressive pulmonary hypertension induced by monocrotaline: a biochemical and ultrastructural study. Lab. Invest. 58:184-195.

6. Ilkiw, R., L. Todorovich-Hunter, K. Maruyama, J. Shin, and M. Rabinovitch. 1989. SC-39026, a serine elastase inhibitor, prevents muscularization of peripheral arteries, suggesting a mechanism of monocrotaline-induced pulmonary hypertension in rats. Circ. Res. 64:814-825.

7. Ye, C., and M. Rabinovitch. 1991. Inhibition of elastolysis by SC-37698 reduces development and progression of monocrotaline pulmonary hypertension. Am. J. Physiol. 261:H1255-H1267.

8. Todorovich-Hunter, L., H. Dodo, C. Ye, L. McCready, F. W. Keeley, and M. Rabinovitch. 1992. Increased pulmonary artery elastolytic activity in adult rats with monocrotaline-induced progressive hypertensive pulmonary vascular disease compared with infant rats with nonprogressive disease. Am. Rev. Respir. Dis. 146:213-223.

9. Shemie, S., and M. Rabinovitch. 1993. The effect of alpha 1-antitrypsin inhibition of early elastase release on the pathophysiology of progressive pulmonary hypertension. Am. Rev. Respir. Dis. 147:A495. (Abstr.)

10. Maruyama, K., C. Ye, M. Woo, H. Venkatacharya, L. D. Lines, M. M. Silver, and M. Rabinovitch. 1991. Chronic hypoxic pulmonary hypertension in rats and increased elastolytic activity. Am. J. Physiol. 261:H1716-H1726.

11. Hornebeck, W., D. Brechemier, J. Soleilhac, M.-C. Bourdillon, and L. Robert. 1985. Studies on rat aorta smooth muscle cells' elastase activity. In Extracellular Matrix: Structure and Function. Vol 25. A. Reddi, editor. Alan R. Liss, Inc., New York. 269-282.

12. Chomczynski, P., and N. Sacchi. 1987. Single-step method of RNA isolation by acid guanidinium thiocyanate-phenol-chloroform extraction. Anal. Biochem. 162:156-159.

13. Sambrook, J., E. F. Fritsch, and T. Maniatis. 1989. Molecular Cloning: A Laboratory Manual. 2nd ed. Cold Spring Harbor Press, Cold Spring Harbor, NY.

14. Min, H. Y., and B. M. Spiegelman. 1986. Adipsin, the adipocyte serine protease: gene structure and control of expression by tumor necrosis factor. $\mathrm{Nu}$ cleic Acids Res. 14:8879-8892.
15. Krishnan, B. R., R. W. Blakesley, and D. E. Berg. 1991. Linear amplification DNA sequencing directly from single phage plaques and bacterial colonies. Nucleic Acids Res. 19:1153.

16. Gassmann, M., P. Thommes, T. Weiser, and U. Hubscher. 1990. Efficient production of chicken egg yolk antibodies against a conserved mammalian protein FASEB (Fed. Am. Soc. Exp. Biol.) J. 4:2528-2532.

17. Hinek, A., D. S. Wrenn, R. S. Mecham, and S. H. Barondes. 1988. The elastin receptor: a galactoside-binding protein. Science (Wash. DC). 239:15391541.

18. Hinek, A., M. Rabinovitch, F. Keeley, Y. Okamura-Oho, and J. Callahan. 1993. The $67-\mathrm{kD}$ elastin/laminin-binding protein is related to an enzymatically inactive, alternatively spliced form of $\beta$-galactosidase. J. Clin. Invest. 91:11981205.

19. Banda, M. J., Z. Werb, and J. H. McKerrew. 1987. Elastin degradation. Methods Enzymol. 144:296-299.

20. Hinek, A., R. P. Mecham, F. Keeley, and M. Rabinovitch. 1991. Impaired elastin fiber assembly related to reduced $67-\mathrm{kD}$ elastin-binding protein in fetal lamb ductus arteriosus and cultured aortic smooth muscle cells treated with chondroitin sulfate. J. Clin. Invest. 88:2083-2094.

21. Flier, J. S., K. S. Cook, P. Usher, and B. M. Spiegelman. 1987. Severely impaired adipsin expression in genetic and acquired obesity. Science (Wash. DC). 237:402-405.

22. Hinek, A., and M. Rabinovitch. 1994. $67-\mathrm{kD}$ elastin-binding protein is a protective "companion" of extracellular insoluble elastin and intracellular tropoelastin. J. Cell Biol. 126:563-574.

23. Cook, K. S., H. Y. Min, D. Johnson, R. J. Chaplinsky, J. S. Flier, C. R. Hunt, and B. M. Spiegelman. 1987. Adipsin: a circulating serine protease homolog secreted by adipose tissue and sciatic nerve. Science (Wash. DC). 237:402-405.

24. Rosen, B. S., K. S. Cook, J. Yaglom, D. L. Groves, J. E. Volanakis, D. W. T. Damm, and B. M. Spiegelman. 1989. Adipsin and complement factor D activity: an immune-related defect in obesity. Science (Wash. DC). 244:14831487.

25. Baldo, A., A. D. Sniderman, A. St-Luce, R. K. Avramoglu, M. Maslowska B. Hoang, J. C. Monge, A. Bell, S. Mulay, and K. Cianflone. 1993. The adipsinacylation stimulation protein system and regulation of intracellular triglyceride synthesis. J. Clin. Invest. 92:1543-1547.

26. Kislauskis, E. H., Z. Li, R. H. Singer, and K. L. Taneja. 1993. Isoformspecific 3'-untranslated sequences sort $\alpha$-cardiac and $\beta$-cytoplasmic actin messenger RNAs to different cytoplasmic compartments. J. Cell Biol. 123:165-172.

27. Foster, J. A., C. B. Rich, and M. F. Miller. 1988. An in vitro model for pulmonary emphysema. J. Cell Biol. 107:597A. (Abstr.)

28. Kobayashi, J., D. Wigle, T. Childs, L. Zhu, F. W. Keeley, and M. Rabinovitch. 1994. Serum-induced vascular smooth muscle cell elastolytic activity through tyrosine kinase intracellular signalling. J. Cell. Physiol. 160:121-131.

29. Oho, S., and M. Rabinovitch. 1994. Post-cardiac transplant arteriopathy in piglets is associated with fragmentation of elastin and increased activity of a serine elastase. Am. J. Pathol. 145:202-210.

30. Oho, S., E. Koo, A. Gotlieb, and M. Rabinovitch. 1992. Increased elastolytic activity associated with intimal proliferation in porcine aortic organ culture. FASEB (Fed. Am. Soc. Exp. Biol.) J. 7:A491. (Abstr.)

31. O'Brien, J., and W. Regan. 1991. A study of elastic tissue and actinic radiation in 'aging' temporal arteritis, polymyalgia rheumatic. The actinic storm in the modern world. $J$. Am. Acad Dermatol. 24:765-776.

32. Lyons, R. M., J. Keski-Oja, and H. L. Moses. 1988. Proteolytic activation of latent transforming growth factors from fibroblast conditioned medium. J. Cell Biol. 106:1660-1665.

33. Sato, Y., and D. B. Rifkin. 1989. Inhibition of endothelial cell movement by pericytes and smooth muscle cells: activation of a latent transforming growth factor- $\beta 1$-like molecule by plasmin during co-culture. J. Cell Biol. 109:309-315.

34. Taipale, J., K. Koli, and J. Keski-Oja. 1992. Release of transforming growth factor- $\beta 1$ from the pericellular matrix of cultured fibroblasts and fibrosarcoma cells by plasmin and thrombin. J. Biol. Chem. 267:25378-25384.

35. Perkett, E. A., R. Lyons, H. Moses, K. Brigham, and B. Meyrick. 1990. Transforming growth factor $\beta$ activity in sheep lung lymph during the development of pulmonary hypertension. J. Clin. Invest. 86:1459-1464.

36. Lindner, V., D. A. Lappi, A. Baird, R. A. Majack, and M. A. Reidy. 1991. Role of basic fibroblast growth factor in vascular lesion formation. Circ. Res. 68:106-113

37. Perkett, E. A., D. B. Badesch, M. K. Roessler, K. R. Stenmark, and B. Meyrick. 1992. Insulin-like growth factor I and pulmonary hypertension induced by continuous air embolization in sheep. Am. J. Respir. Cell Mol. Biol. 6:82-87. 38. Dempsey, E. C., K. R. Stenmark, I. F. McMurtry, R. F. O'Brien, N. F. Voelkel, and D. B. Badesch. 1990. Insulin-like growth factor I and protein kinase C activation stimulate pulmonary artery smooth muscle cell proliferation through separate but synergistic pathways. J. Cell. Physiol. 14:159-165. 